\title{
POLG gene mutation. Clinico-neuropathological study
}

\author{
Sylwia Tarka ${ }^{1}$, Milena Laure-Kamionowska², Teresa Wierzba-Bobrowicz ${ }^{3}$, Katarzyna Witulska ${ }^{4}$, Elżbieta Ciara ${ }^{5}$, \\ Krystyna Szymańska ${ }^{2}$, Paweł Krajewski ${ }^{1}$, Tomasz Stępieñ ${ }^{3}$, Albert Acewicz ${ }^{3}$, Paulina Felczak ${ }^{3}$ \\ ${ }^{1}$ Department of Forensic Medicine, Medical University of Warsaw, Warsaw, Poland, ${ }^{2}$ Department of Experimental and Clinical \\ Neuropathology, Mossakowski Medical Research Centre, Polish Academy of Sciences, Warsaw, Poland, ${ }^{3}$ Department of Neuropathology, \\ Institute of Psychiatry and Neurology, Warsaw, Poland, ${ }^{4}$ Department of Intensive Care, The Children's Memorial Health Institute (CMHI), \\ Warsaw, Poland, ${ }^{5}$ Department of Medical Genetics, The Children's Memorial Health Institute (CMHI), Warsaw, Poland
}

\begin{abstract}
We present a female patient with a mutation of the POLG gene (POLG DNA polymerase gamma, catalytic subunit; *174763) in which the clinical course suggested a mitochondrial disease, a neuropathological examination identified the syndrome more closely, and a genetic test confirmed the disease. Apart from the morphological lesions typical of Alpers-Huttenlocher syndrome, rarely observed symmetrical degenerative changes in the accessory olivary nuclei were found. It was unusual in the clinical course of the disease that pancreatitis was diagnosed before symptoms of liver failure appeared.
\end{abstract}

Key words: POLG-mutation, Alpers-Huttenlocher syndrome, acute pancreatitis, medial accessory olivary nuclei.

\section{Introduction}

The term mitochondrial diseases refers to a group of disorders related to the respiratory chain and oxidative phosphorylation (OXPHOS) dysfunction [27]. The genetic and clinical heterogenity of these diseases make their diagnosis difficult, the statistics underestimated, and the pathogenesis still unclear [17]. In mitochondrial encephalopathies, central nervous system structures are affected according to different patterns of distribution and severity. The changes in the brain can be restricted to specific areas or be diffuse, involving the entire cerebrum $[6,8]$. Characteristic lesions are neuronal loss, vasculo-necrotic changes, gliosis, demyelination and spongy degeneration $[8,20]$.
Despite the significant overlap of the clinical and neuropathological picture between the various mitochondrial diseases, in a few cases the neuropathological picture is sufficiently characteristic to suggest a diagnosis of Leigh syndrome or MELAS [5]. One of the mitochondrial diseases with an uncharacteristic and varied neuropathological picture is the Alpers-Huttenlocher syndrome - progressive neuronal degeneration with liver failure [1,15,22].

Mutations in the nuclear gene POLG are an important cause of mitochondrial diseases. The POLG gene encodes the mitochondrial DNA (mtDNA) polymerase gamma catalytic subunit $\left({ }^{*} 174763\right)$ that is responsible for replication and repair of the mitochondrial genome. Loss of mtDNA leads to loss of mitochondrial respiratory chain components, what, 
in turn, progressively disables energy metabolism $[7,19]$.

POLG defects result in syndromes of mitochondrial DNA depletion or accumulation of mitochondrial DNA deletions. Defects in this gene are a cause of progressive external ophthalmoplegia with mitochondrial DNA deletions 1 (PEOA1), sensory ataxic neuropathy dysarthria and ophthalmoparesis (SANDO), Alpers-Huttenlocher syndrome (AHS), and mitochondrial neurogastrointestinal encephalopathy syndrome (MNGIE) [19,23,31,34].

Alpers' syndrome is one of the most severe phenotypes, characterized by intractable epilepsy, developmental regression and liver failure which affects children aged 6 months to 3 years. The later onset variants are mainly characterized by epileptic encephalopathy with ataxia.

The presented patient is typical with regard to the first clinical symptoms such as epilepsy and developmental regression, but unusual for the further course of the disease.

\section{Clinico-morphological presentation}

The girl was born as one of twins. Her psychomotor development was normal up to the age of 2. In preschool age, the girl had problems with adapting to her peer environment. There were learning problems at school, she repeated the first grade. The psychological examination revealed a lower than average mental level, underdeveloped phonemic awareness and visual perception, poor spatial orientation and the recognition of the body schema. From the age of six, focal and generalized myoclonus and less agile gait appeared. At the age of 8 developmental regression was observed.
She was hospitalized due to myoclonic seizures in the department of neurology at the age of 8 . No neurological syndrome was found in the neurological examination. Magnetic resonance imaging was normal. There was an abnormal electroencephalography with localized and generalized epileptiform activity. The diagnosis was epilepsy with myoclonic seizures. Treatment with valproic acid was started.

Three months later the girl was re-admitted to the hospital with a sudden onset of abdominal pain, diarrhoea and vomiting. An abdominal ultrasound revealed an enlarged pancreas with blurred margins, no changes in the liver were found, the diagnosis was acute pancreatitis. The child's condition deteriorated rapidly - 3 days later she developed circulatory and respiratory insufficiency, jaundice, and symptoms of haemorrhagic diathesis. The diagnosis was acute hepatic failure of unclear aetiology with progressive multiple organ failure. Wilson's disease was ruled out. Mitochondrial disease was suspected and genetic testing was done. The girl died before the completion of diagnosis, on the $5^{\text {th }}$ day after the onset abdominal symptoms.

\section{Pathomorphological examination}

The post-mortem examination showed jaundice, features of a haemorrhagic diathesis, fluid in the peritoneal and pleural cavities and blurred margins of the liver and pancreas. The microscopic examination of the pancreas revealed: diffuse, massive parenchymal necrosis with inflammatory infiltrates mainly consisting of granulocytes, and haemorrhages (Fig. 1A). In the liver necrosis of the central lobule, cholestasis, small droplet steatosis, and mixed
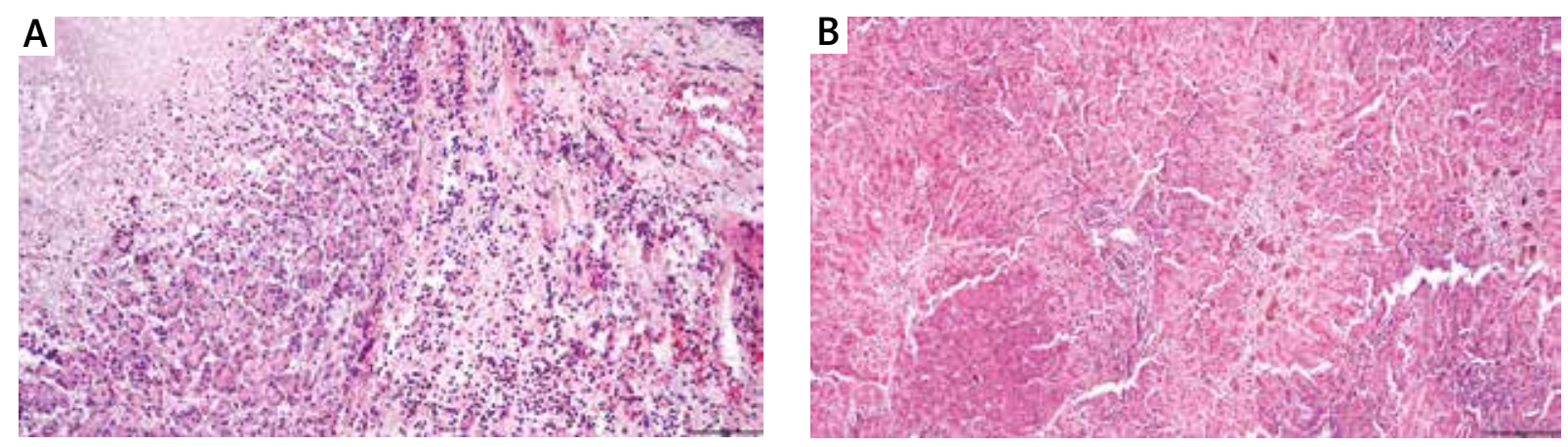

Fig. 1. A) Massive pancreatic necrosis with acute inflammatory infiltration (H\&E 20x); B) Focal liver necrosis with small inflammatory infiltration and cholestasis (H\&E 10x). 


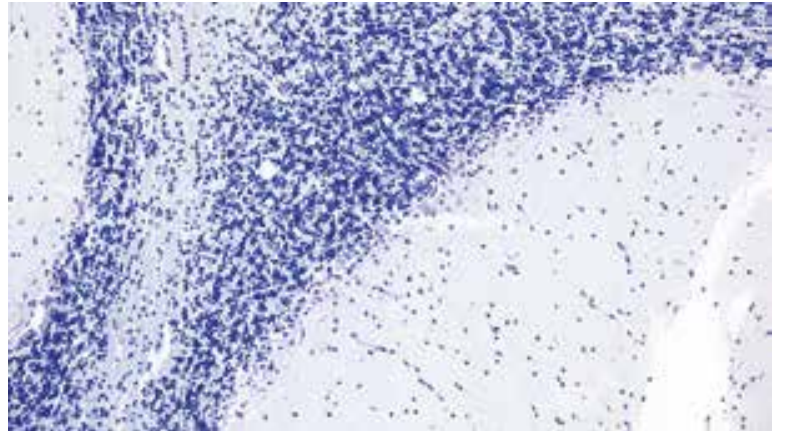

Fig. 2. Cerebellum. Loss of Purkinje cells with proliferation of Bergman's glia (Cresyl violet 20x).

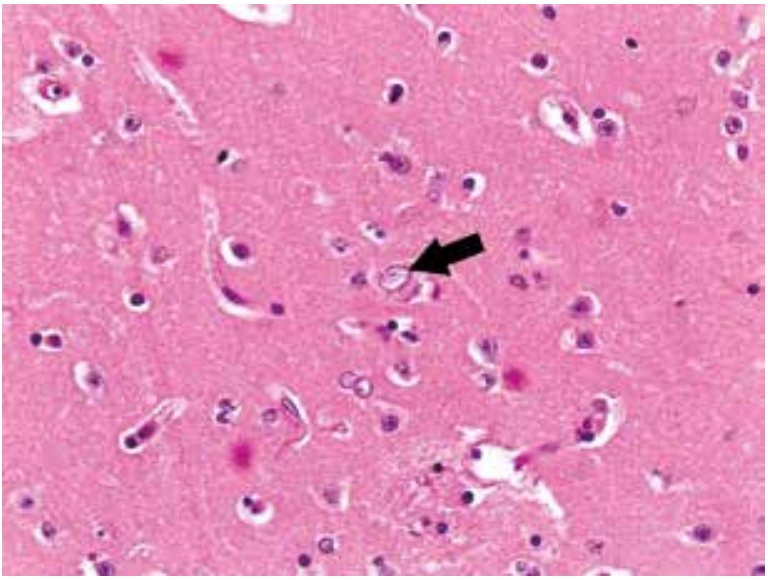

Fig. 4. Basal ganglia. Alzheimer's type II cells (H\&E 40x).

inflammatory infiltrates consisting of lymphocytes and individual granulocytes were observed (Fig. 1B).

\section{Neuropathological examination}

The material derived from the First Polish Brain Bank in the Institute of Psychiatry and Neurology, Warsaw, Poland.

Macroscopically the brain was swollen with reduced consistency of the basal ganglia and pale substantia nigra.

Microscopic examination was performed on paraffin embedded slides from the cerebral hemispheres, the brain stem and the cerebellum. The sections were stained with haematoxylin eosin, cresyl violet, Klüver-Barrera method, methods for iron (Pearse) and copper (method using Rhodamine B) and immunohistochemically with GFAP (DAKO $1: 70$ ), Ubiquitin (Invitrogen 1 : 90), Tau (DAKO $1: 80$ ), and $\alpha$-synuclein (Leica 1 : 30).

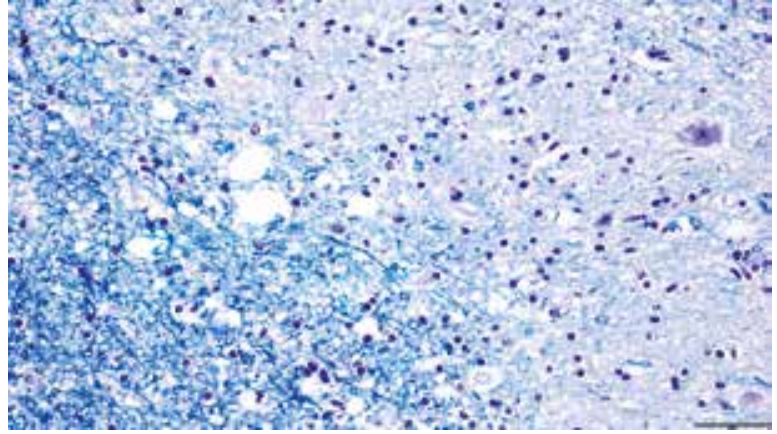

Fig. 3. Demyelination in the cerebellum (dentate nucleus) (Klüver-Barrera 40x).

The microscopic evaluation revealed neuronal damage in the striatum, thalamus, dentate nucleus, substantia nigra, red nuclei, and inferior olivary nuclei. Neurons with peripherally localized chromatin, vacuoles under the cytoplasmic membrane and neuronal shadows were observed. In the cerebral hemispheres, disseminated cortical lesions were found. Widespread necrosis within layers II and IV with moderate glia reaction and spongiosis was visible in many cortical gyri. In the cerebellum, damage of neurons was found in the dentate nuclei. Dispersed loss of Purkinje cells with proliferation of Bergman's glia was demonstrated (Fig. 2). Focal demyelination, accompanied by mild spongiosis was observed in both hemispheres of the brain and cerebellum (Fig. 3). Myelin showed fading especially in the central part of the cerebral hemispheres and the cerebellum, but the subcortical fibres were spared. In the basal ganglia, few type II Alzheimer's glial cells were found (Fig. 4). In the medulla the hypertrophic medial accessory olivary nuclei filled with numerous gemistocytic and bizarre glial cells were visible (Fig. 5). This lesion was found symmetrically and bilaterally in the accessory olivary nuclei. The neurons of the substantia nigra showed positive reaction to ubiquitin, indicating their damage. No positive immunoreactivity to tau and synuclein was observed. Pathological copper or iron deposition was not found by microscopic examination.

\section{Genetic examination}

DNA test results were obtained after the child died. A genetic test revealed biallelic POLG gene mutations. The WES study showed that the girl was a heterozygous carrier of two known molecular variants in the POLG gene (RefSeq: NM_002693.2; 
A
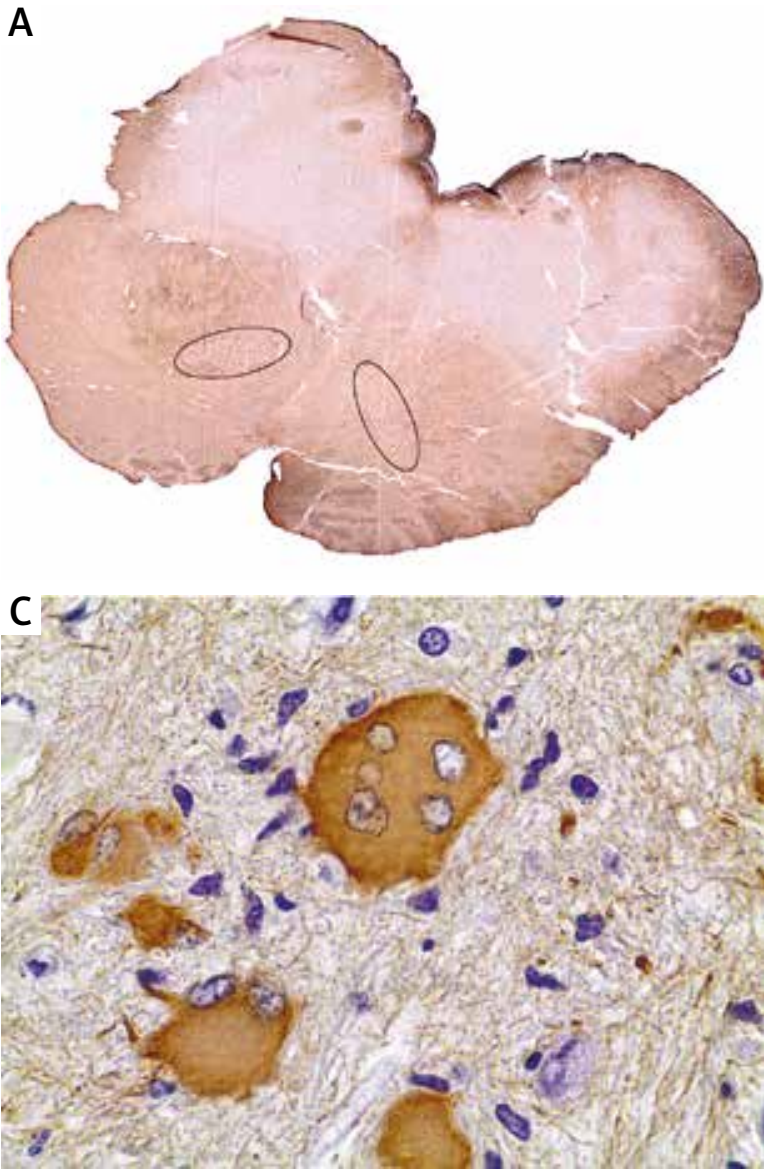
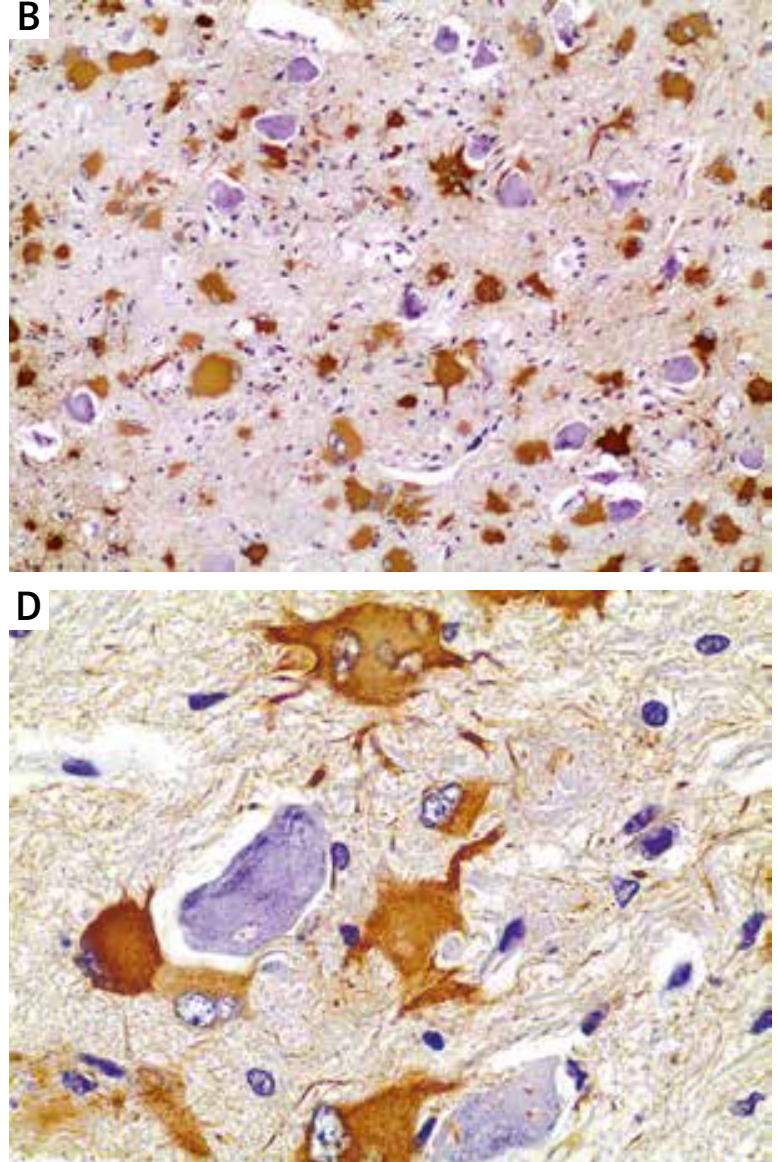

Fig. 5. Medulla oblongata, medial accessory olivary nucleus. A) Symmetrically hypertrophic accessory olivary nuclei (GFAP 4x); B) Numerous reactive astrocytes (GFAP 20x); C, D) Multinuclear, bizarre astrocytes (GFAP 60x).

NP_002684.1): c.2243G>C p.(Trp748Ser) and c.2542G>A p.(Gly848Ser). The clinical significance of changes in the POLG gene was reported in the OMIM database (Online Mendelian Inheritance in Man) in the context of the phenotypes: $4 \mathrm{~A}$ mitochondrial depletion syndrome (Alpers type, \#203700), 4B mitochondrial depletion syndrome (MNGIE type, \#613662), Ataxia syndrome (including SANDO and SCAE, \#607459), and progressive external ophthalmoplegia (PEOB1, \#258450).

\section{Discussion}

In the presented case, the clinical symptoms (myoclonic epilepsy, regression) were not sufficient to diagnose mitochondrial disease, but it could be suspected. The occurrence of acute hepatic failure shortly after the treatment by valproic acid also very strongly indicated a mitochondrial disease, in partic- ular Alpers-Huttenlocher syndrome [13]. The neuropathological examination revealed neuronal degeneration in the cortex of the brain with spongiosis and gliosis, but these lesions were of a low intensity. The neuronal lesions were also found in the cerebellar cortex, dentate nucleus and slight demyelination was observed in the cerebellar white matter. Neuronal damage was also seen in the striatum, thalamus, substantia nigra, red nuclei and inferior olivary nuclei. The specific changes in the form of neuronal degeneration with presence of bizarre, hypertrophic astroglial cells were found symmetrically within the medulla oblongata. The spectrum of morphological changes is consistent with those described in Alpers-Huttenlocher syndrome $[8,12,13,28]$. In the striatum there were a few pathological Alzheimer's type II glial cells similar to presented by Simonati in a few Alpers-Huttenlocher syndrome cases [28]. 
Alzheimer's type II cells, although typical of liver failure (hepatic encephalopathy), are rarely described in Alpers-Huttenlocher syndrome.

Hayhurst et al. demonstrated extensive involvement of occipital cortical interneurons and pyramidal neurons and reduced density of cerebellar Purkinje cells in patients with Alpers syndrome. The authors showed evidence of extensive complex I defects affecting GABAergic cortical interneurons and cerebellar Purkinje cells. Authors suggested that interneuron and Purkinje cell pathology underlies the development of seizures and ataxia in Alpers' syndrome [14].

It was unusual in the clinical course of the disease that pancreatitis was diagnosed before symptoms of liver failure appeared. Acute pancreatitis caused by valproic acid is reported extremely rarely (especially in children) and does not have to be associated with mitochondrial disease by some authors $[2,10,29]$. However, other authors underlie that pancreatitis can be an unusual complication associated with mitochondrial disease. Toyono reported the first case of chronic pancreatitis associated with mitochondrial encephalopathy with the m.8344A>G in MTTK mitochondrial gene [33]. Ishiyama described 2 cases of mitochondrial disease complicated by pancreatitis as an unusual pancreatic exocrine manifestation [16]. Montine presented Alpers' syndrome with seizures and multiple stroke-like episodes and acute haemorrhagic pancreatitis [21]. Finsterer and Frank reviewed gastrointestinal manifestations of mitochondrial disorders and pancreatitis with or without diabetes and with or without exocrine pancreas insufficiency. Authors underlined that the cause of pancreatitis in mitochondrial diseases is unknown, but it can be speculated that the metabolic defect in exocrine pancreas cells promotes a defect in exocrine enzyme secretion, which could result in intracellular protease accumulation and activation [9].

In the presented case, the symmetrical bilateral damage of the medial accessory olivary nuclei is worth noting. The olivary nuclei became hypertrophic, with bizarre, deformed nerve cells and many multinuclear astrocytes. Hypertrophic olivary degeneration is a rare form of trans-synaptic degeneration and has been reported in inferior olivary nucleus. Injury to the dentato-rubro-olivary pathway causes hypertrophy and enlargement of the inferior olivary nuclei, which is called hypertrophic olivary degeneration [26]. It can be observed in ischemic stroke, neoplasms, pons haemorrhage, vascular malformations damaging the dentato-olivary pathway [32]. Medial accessory nucleus is a part of the accessory olive-cerebellar pathway, and the damage of this pathway corresponds to the ataxia.

Goto et al. revealed six different stages of olivary changes after the destruction of the central tegmental tract in the pons: 1) no olivary changes, 2) olivary fibres degeneration, 3) olivary hypertrophy, 4) culminant olivary enlargement, 5) olivary pseudohypertrophy, and 6) olivary atrophy. In fourth and fifth stages prominent gemistocytic astrocytes in the characteristically enlarged inferior olivary nuclei were found [11]. In our case, the intensive gemistocytic, multinuclear astrocytic reaction was observed within the olivary nucleus, what suggested a longer-lasting degeneration process.

These secondary degenerative changes were described mainly in the inferior olivary nucleus. Retrograde degeneration within the medial accessory olivary nucleus has been reported very rarely. It was presented by Robertson in association with chronic cerebellar stimulation [25].

Bilateral hypertrophic olivary degeneration, but only on brain MRI, has been reported in a few metabolic, genetic and neurodegenerative disorders, including mitochondrial disorders such as Leigh and Leigh-like syndrome $[4,30]$ or in patients with heterozygous mutations in the POLG gene [18]. It should be mentioned here that there is a difference in the diagnosis of hypertrophic olivary degeneration by radiologists and neuropathologists. In the quoted articles on hypertrophic olivary degeneration in MRI, authors described enlargement, lateral placement, round shape and focal T2 hyperintensity as the criteria defining hypertrophic olivary degeneration $[4,18,30]$. In the histopathological evaluation, the term hypertrophic olivary degeneration refers mainly to degenerative changes within the olivary nucleus [11]. It should be emphasized that the radiological diagnosis of hypertrophic olivary degeneration is not strictly consistent with the histopathological diagnosis and the use of pathological diagnosis to describe radiological changes may be confusing.

There is great variability in the clinical course and the neuropathological picture in cases of POLG gene mutation and it remains unclear what causes this diversity. Betts suggests that this is due to a small number of autopsies performed, especially those 
which include a complete neuropathological examination [3].

The most common POLG mutation, p.Ala467Thr also exhibits considerable phenotypic heterogeneity. Rajakulendran et al. analysed this mutation in mitochondrial diseases. Authors evaluated the clinical, neuropathological and mitochondrial genetic features in unrelated patients with homozygous p.Ala 467Thr mutations and considered the mechanisms by which homozygous p.Ala467Thr mutations give rise to such diverse phenotypes. Their conclusions emphasize the variable and overlapping clinical and neuropathological phenotypes and molecular defects caused by the A467T mutation, which may result from factors such as the mtDNA genetic background, nuclear genetic modifiers and environmental stressors [24].

Accurate diagnosis, including post-mortem, of each case of Alpers-Huttenlocher syndrome is valuable not only for genetic counselling, but also because of the need for further research into the pathogenesis of this syndrome and other mitochondrial diseases.

\section{Acknowledgements}

Funding sources: The study was partially (molecular genetics) funded by The Children's Memorial Health Institute intramural grant S245/17.

This work is supported by the "Digital Brain digital collection of the Institute of Psychiatry and Neurology" (Project No. POPC.02.03.01-00.0042/18$00)$. The "Digital Brain" project is co-funded by the European Union and the Polish budget. The authors are very grateful to the First Polish Brain Bank in the Institute of Psychiatry and Neurology, Warsaw, Poland.

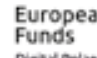

Funds

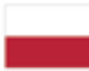

Republic

of Poland

European Union

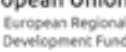

\section{References}

1. Alpers BJ. Diffuse progressive degeneration of the gray matter of the cerebrum. Arch Neurol Psychiatry 1931; 25: 469-505; after Gray F, Keohane K, Adele-Biassette H, Harding BN, Golden JA. Developmental neuropathology. $2^{\text {nd }}$ ed. Mitochondrial Disorders 2018; 398-399.

2. Batalden PB, Van Dyne BJ, Cloyd J. Pancreatitis associated with valproic acid therapy. Pediatrics 1979; 64: 520-552.

3. Betts J, Lightowlers RN, Turnbull DM. Neuropathological aspects of mitochondrial DNA disease. Neurochem Res 2004; 29: 505-511.
4. Bindu PS, Taly AB, Sonam K, Govindaraju C, Arvinda HR, Gayathri N, Bharath MM, Ranjith D, Nagappa M, Sinha S, Khan NA, Thangaraj K. Bilateral hypertrophic olivary nucleus degeneration on magnetic resonance imaging in children with Leigh and Leigh-like syndrome. Br J Radiol 2014; 87: 20130478.

5. Brown GK Squier MV. Neuropathology and pathogenesis of mitochondrial diseases. J Inherit Metab Dis 1996; 19: 553-572.

6. Brown GK. Congenital brain malformations in mitochondrial disease. J Inherit Metab Dis 2005; 28: 393-401.

7. Cohen BH, Chinnery PF, Copeland WC. POLG-Related Disorders. 2010 Mar 16 [updated 2018 Mar 1]. In: Adam MP, Ardinger HH, Pagon RA, Wallace SE, Bean LJH, Stephens K, Amemiya A (Eds.). GeneReviews $^{\circledR}$ [Internet]. University of Washington, Seattle 1993-2020. PMID: 20301791.

8. Filosto M, Tomelleri G, Tonin P, Scarpelli M, Vattemi G, Rizzuto N, Padovani A, Simonati A. Neuropathology of mitochondrial diseases. Biosci Rep 2007; 27: 23-30.

9. Finsterer J, Frank M. Gastrointestinal manifestations of mitochondrial disorders: a systematic review. Therap Adv Gastroenterol 2017; 10: 142-154.

10. Gerstner T, Büsing D, Bell N, Longin E, Kasper JM, Klostermann W, HebingB, HanefeldF,EckelU, HoffmannR, BettendorfU, WeidnerB, Wiemer-Kruel A, Brockmann K, Neumann FW, Sandrieser T, Wolff M, König S. Valproic acid-induced pancreatitis: 16 new cases and a review of the literature. J Gastroenterol 2007; 42: 39-48.

11. Goto N, Kaneko M. Olivary enlargement: Chronological and morphometric analyses. Acta Neuropathol 1981; 54: 275-282.

12. Harding BN, Egger J, Portmann B, Erdohazi M. Progressive neuronal degeneration of childhood with liver disease. A pathological study. Brain 1986; 109: 181-206.

13. Harding BN. Progressive neuronal degeneration of childhood with liver disease (Alpers-Huttenlocher syndrome): a personal review. J Child Neurol 1990; 5: 273-287.

14. Hayhurst H, Anagnostou ME, Bogle HJ, Grady JP, Taylor RW, Bindoff LA, McFarland R, Turnbull DM, Lax NZ. Dissecting the neuronal vulnerability underpinning Alpers' syndrome: a clinical and neuropathological study. Brain Pathol 2019; 29: 97-113.

15. Huttenlocher PR, Solitare GB, Adams G. Infantile diffuse cerebral degeneration with hepatic cirrhosis. Arch Neurol 1976; 33: 186-192.

16. Ishiyama A, Komaki H, Saito T, Saito Y, Nakagawa E, Sugai K, Itagaki Y, Matsuzaki K, Nakura M, Nishino I, Goto Y, Sasaki M. Unusual exocrine complication of pancreatitis in mitochondrial disease. Brain Dev 2013; 35: 654-659.

17. Khan NA, Govindaraj P, Meena AK, Thangaraj K. Mitochondrial disorders: challenges in diagnosis \& treatment. Indian J Med Res 2015; 141: 13-26.

18. Kinghorn KJ, Kaliakatsos M, Blakely EL, Taylor RW, Rich P, Clarke A, Omer S. Hypertrophic olivary degeneration on magnetic resonance imaging in mitochondrial syndromes associated with POLG and SURF1 mutations. J Neurol 2013; 260: 3-9.

19. Longley MJ, Graziewicz MA, Bienstock RJ, Copeland WC. Consequences of mutations in human DNA polymerase gamma. Gene 2005; 354: 125-131.

20. Mierzewska H. Choroby mitochondrialne - diagnostyka i postępowanie. Neurologia po Dyplomie 2017; 1: 1-9. 
21. Montine TJ, Powers JM, Vogel FS, Radtke RA. Alpers' syndrome presenting with seizures and multiple stroke-like episodes in a 17-year-old male. Clin Neuropathol 1995; 14: 322-326.

22. Naviaux RK, Nyhan WL, Barshop BA, Poulton J, Markusic D, Karpinski NC, Haas RH. Mitochondrial DNA polymerase gamma deficiency and mtDNA depletion in a child with Alpers' syndrome. Ann Neurol 1999; 45: 54-58.

23. Rahman S, Copeland WC. POLG-related disorders and their neurological manifestations. Nat Rev Neurol 2019; 15: 40-52.

24. Rajakulendran S, Pitceathly RD, Taanman JW, Costello H, Sweeney MG, Woodward CE, Jaunmuktane Z, Holton JL, Jacques TS, Harding BN, Fratter C, Hanna MG, Rahman S. A clinical, neuropathological and genetic study of homozygous A467T POLG related mitochondrial disease. PLoS One 2016; 11: e0145500.

25. Robertson LT, Dow RS, Cooper IS, Levy LF. Morphological changes associated with chronic cerebellar stimulation in the human. J Neurosurg 1979; 51: 510-520.

26. Sanverdi SE, Oguz KK, Haliloglu G. Hypertrophic olivary degeneration in children: four new cases and a review of the literature with an emphasis on the MRI findings. Br J Radiol 2012; 85: 511-516.

27. Schapira AH. Mitochondrial disease. Lancet 2006; 368: 70-82.

28. Simonati A, Filosto M, Savio C, Tomelleri G, Tonin P, Dalla Bernardina B, Rizzuto N. Features of cell death in brain and liver, the target tissues of progressive neuronal degeneration of childhood with liver disease (Alpers-Huttenlocher disease). Acta Neuropathol 2003; 106: 57-65.

29. Sinclair DB, Berg M, Breault R. Valproic acid-induced pancreatitis in childhood epilepsy: case series and review. J Child Neuro 2004; 19: 498-502.

30. Sonam K, Khan NA, Bindu PS, Taly AB, Gayathri N, Bharath MM, Govindaraju C, Arvinda HR, Nagappa M, Sinha S, Thangaraj K. Clinical and magnetic resonance imaging findings in patients with Leigh syndrome and SURF1 mutations. Brain Dev 2014; 36: 807-812

31. Stumpf JD, Saneto RP, Copeland WC. Clinical and molecular features of POLG-related mitochondrial disease. Cold Spring Harb Perspect Biol 2013; 5: a011395.

32. Tilikete C, Desestret V. Hypertrophic olivary degeneration and palatal or oculopalatal tremor. Front Neurol 2017; 8: 302.

33. Toyono M, Nakano K, Kiuchi M, Imai K, Suzuki H, Shishikura K, Osawa M, Shiratori K, Goto Y, Nonaka I, Sugie H. A case of MERRF associated with chronic pancreatitis. Neuromuscul Disord 2001; 11: 300-304.

34. Wong LJ, Naviaux RK, Brunetti-Pierri N, Zhang Q, Schmitt ES Truong C, Milone M, Cohen BH, Wical B, Ganesh J, Basinger AA, Burton BK, Swoboda K, Gilbert DL, Vanderver A, Saneto RP, Maranda B, Arnold G, Abdenur JE, Waters PJ, Copeland WC. Molecular and clinical genetics of mitochondrial diseases due to POLG mutations. Hum Mutat 2008; 29: E150-172. 